\title{
Successful management of diastolic heart failure in a patient with restrictive cardiomyopathy following an anterior communicating artery aneurysm clipping
}

\author{
Rajasekar Arumugam, Georgene Singh, Krishnaprabhu Raju', Ramamani Mariappan
}

\begin{abstract}
Perioperative management of neurosurgical patients with an underlying myocardial dysfunction poses a unique challenge to the neuroanaesthesiologist and the neurointensivist. Sudden catecholamine surge during the aneurysmal subarachnoid haemorrhage (SAH) can cause severe cardiac dysfunction such as myocardial ischaemia and cardiomyopathy. SAH in a patient with restrictive cardiomyopathy could be hazardous to the myocardium leading to severe cardiac morbidity. We report the successful management of an acute post-operative diastolic heart failure with a milrinone infusion in a patient with restrictive cardiomyopathy, following anterior communicating artery aneurysm clipping. We have discussed the 'dual beneficial' role of milrinone on the heart and the brain in this clinical setting. In addition, the importance of peri-operative utilisation of transoesophageal echocardiography and transthoracic echocardiography for the successful management of diastolic heart failure is highlighted.
\end{abstract}

Key words: Anterior communicating artery aneurysm, diastolic heart failure, milrinone, restrictive cardiomyopathy, subarachnoid haemorrhage, transoesophageal, transthoracic echocardiography

\section{INTRODUCTION}

Perioperative management of neurosurgical patients with an underlying myocardial dysfunction poses a unique clinical challenge. The sudden surge of catecholamine during aneurysmal subarachnoid haemorrhage $(\mathrm{SAH})$ can cause cardiac abnormalities ranging from arrhythmia, subendocardial ischaemia and cardiomyopathy (Takotsubo). The presence of both underlying cardiac disease and cardiac dysfunction resulting from the SAH adds to the cardiac morbidity. The perioperative haemodynamic fluctuations, fluid

Departments of Anaesthesia and ${ }^{1}$ Neurological Sciences, Christian Medical College, Vellore, Tamil Nadu, India

Address for correspondence:

Dr. Ramamani Mariappan, Department of Anaesthesia,

Christian Medical College, Vellore, Tamil Nadu, India.

E-mail: ramamani@cmcvellore.ac.in

\begin{tabular}{|l|l|}
\hline \multicolumn{2}{|c|}{ Access this article online } \\
\hline Quick Response Code: & Website: \\
\hline & www.jnaccjournal.org \\
\cline { 2 - 2 } & \\
\hline
\end{tabular}

management and the complications of SAH such as vasospasm and its management, all could be hazardous to the myocardium with limited reserve. Diastolic heart failure contributes to nearly $50 \%$ of heart failure admissions and is often difficult to diagnose and very challenging to manage. ${ }^{[1]}$ We report the successful management of a patient with restrictive cardiomyopathy with Grade III diastolic dysfunction with paroxysmal atrial fibrillation for anterior communicating (Acom) artery clipping.

\section{CASE REPORT}

A 67-year-old hypertensive, asthmatic, and an obese male was planned for Acom artery aneurysm $(13 \mathrm{~mm} \times 11 \mathrm{~mm}$ anteroposterior and transverse diameter with the narrow neck) clipping. One month before this admission, he was admitted elsewhere with SAH and cardiac failure;

This is an open access article distributed under the terms of the Creative Commons Attribution-NonCommercial-ShareAlike 3.0 License, which allows others to remix, tweak, and build upon the work non-commercially, as long as the author is credited and the new creations are licensed under the identical terms.

For reprints contact: reprints@medknow.com

How to cite this article: Arumugam R, Singh G, Raju K, Mariappan R. Successful management of diastolic heart failure in a patient with restrictive cardiomyopathy following an anterior communicating artery aneurysm clipping. J Neuroanaesthesiol Crit Care 2017;4:120-3. 
evaluated and found to have ruptured Acom artery aneurysm and restrictive cardiomyopathy with Grade III diastolic dysfunction. He was managed medically and sent to our hospital for further management. During his pre-operative assessment, his Glasgow Coma Scale (GCS) score was 15/15 and he gave a history of exertional dyspnoea, reduced functional capacity (<3 METs) and the symptoms of obstructive sleep apnoea. On general examination, he was morbidly obese with the body mass index of $37 \mathrm{~kg} / \mathrm{m}^{2}$ and had several predictors of difficult airway including high Mallampati score (Grade III), prognathism and moderate limitation of neck extension. He presented with paroxysmal atrial fibrillation with the controlled ventricular rate on carvedilol $(3.125 \mathrm{mg}$ ) and was also on ramipril $(2.5 \mathrm{mg})$ for his hypertension. Despite a high $\mathrm{CHA}_{2} \mathrm{DS}_{2}-\mathrm{VASc}$ score (score of 4) with increased risk for stroke, he was not started on anticoagulants, given his recent aneurysmal bleed. On cardiovascular examination, his vitals were stable with blood pressure (BP) of $140 / 80 \mathrm{mmHg}$ and heart rate of 55 beats/ $\mathrm{min}$, and there were no clinical signs of cardiac failure. His respiratory and neurological examinations were within normal limits, the pre-operative investigations were within normal limits and the electrocardiogram (ECG) showed sinus rhythm with the first-degree atrioventricular block with premature ventricular complex and the ' $q$ ' waves in inferolateral leads [Figure 1]. Echocardiography revealed restrictive cardiomyopathy with Grade III diastolic dysfunction and a normal systolic function. Given his medical comorbidities, a high-risk consent was obtained.

In the operating room, standard monitors (5-lead ECG, $\mathrm{SpO}_{2}$ and non-invasive $\mathrm{BP}$ ) were established. A 16-gauge peripheral venous cannula and left radial arterial line were secured under local anaesthesia. The patient was pre-oxygenated for 5 min with $100 \%$ oxygen, and anaesthesia was induced with etomidate $(0.2 \mathrm{mg} /$ $\mathrm{kg})$, fentanyl $(2 \mathrm{mcg} / \mathrm{kg})$ and sevoflurane. Muscle

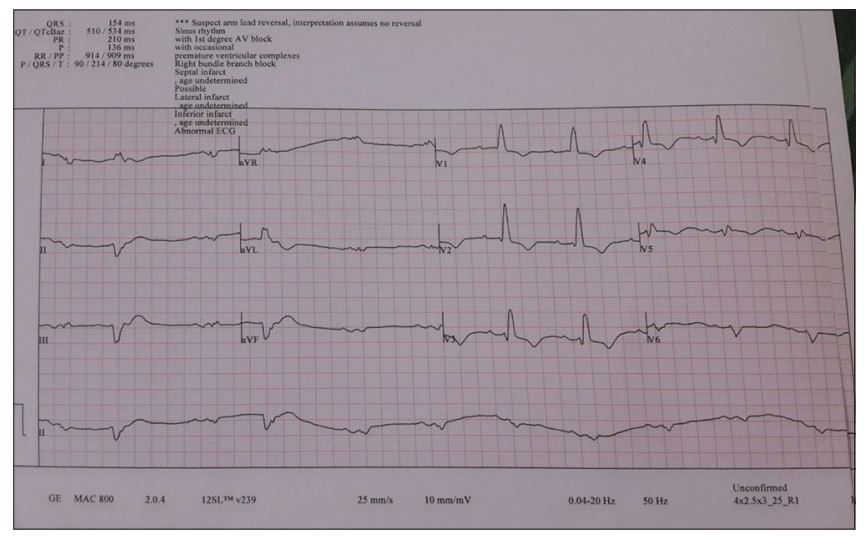

Figure 1: Patient's pre-operative electrocardiogram showing sinus rhythm with the first-degree atrioventricular block with premature ventricular complex and the ' $q$ ' waves in inferolateral leads relaxation was achieved with succinylcholine $(1.5 \mathrm{mg} /$ $\mathrm{kg}$ ) and intubated with $8.5 \mathrm{~mm}$ endotracheal tube using glidescope (British Columbia, Canada). Intubation response was blunted with intravenous (IV) xylocaine (100 mg) and fentanyl $(50 \mu \mathrm{g})$. Right subclavian central venous catheter was inserted for infusion of vasopressors and inotropes. Temperature and end-tidal carbon dioxide and the agent analyser, somatosensory-evoked potential (SSEP) and TOF monitors were established. Anaesthesia was maintained with air, oxygen and 0.8-0.9 minimum alveolar concentration desflurane, and intermittent doses of fentanyl were administered for pain. Neuromuscular paralysis was maintained with $5-7 \mathrm{mcg} / \mathrm{kg} / \mathrm{min}$ of atracurium. Scalp block was given using $20 \mathrm{ml}$ of $0.2 \%$ ropivacaine and $10 \mathrm{ml}$ of $2 \%$ xylocaine. Keeping the baseline diastolic dysfunction and the need for optimal fluid to prevent vasospasm in aneurysmal surgery, trans-oesophageal echocardiography (TEE) probe was inserted. Baseline recordings revealed a mitral Doppler inflow velocity ratio E/A $>2.5$ with prominent ' $\mathrm{D}$ ' waves in the pulmonary venous Doppler flow consistent with Grade III diastolic dysfunction as shown in Figures 2 and 3.

Considering the Grade III diastolic dysfunction and atrial fibrillation, the patient was started on milrinone infusion $(0.5 \mathrm{mcg} / \mathrm{kg} / \mathrm{min})$ without the bolus dose. A low dose of noradrenaline infusion $(0.03-0.05 \mathrm{mcg} / \mathrm{kg} / \mathrm{min})$ was begun to counteract the hypotension associated with the induction of anaesthesia, to avoid excessive fluid administration and to maintain the BP within the normal range. The heart rate and mean arterial pressure were maintained within $15 \%$ of baseline value. The pre-load and inotropic requirements were tailored by the TEE

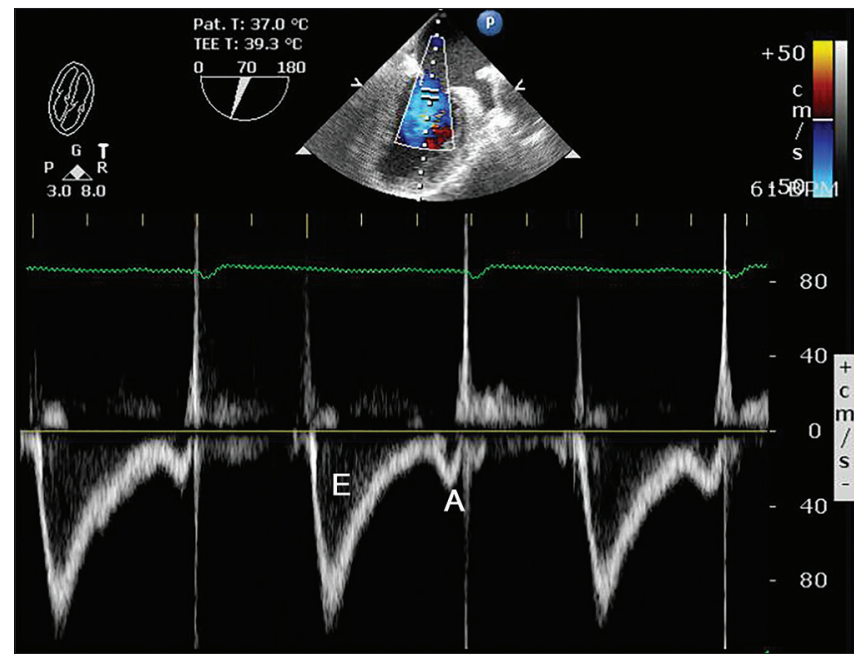

Figure 2: Trans-oesophageal transmitral Doppler flow trace illustrating the prominent peak $E$ (early diastolic) wave velocity in comparison with peak $A$ (atrial) wave velocity and the E/A ratio $>2$. E: Early diastolic wave, A: Atrial wave (mid-oesophageal 2-chamber view with left atrial appendage) 


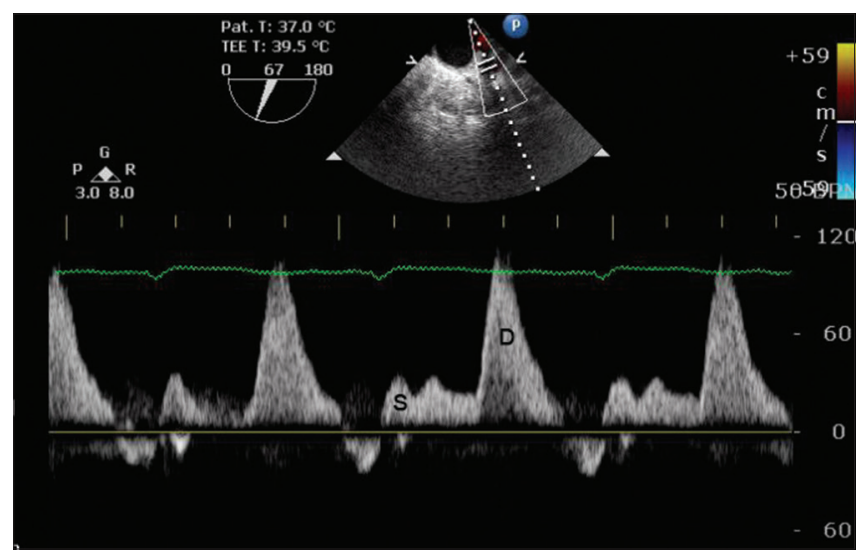

Figure 3: Trans-oesophageal pulmonary venous Doppler trace showing the dampened $S$ (peak systolic velocity wave) with prominent $D$ (diastolic) wave trend: $S<D$ ratio. S: Systolic wave, D: Diastolic wave (mid-oesophageal $60^{\circ}$ view of the left upper pulmonary vein)

assessment. A pterional craniotomy was performed, and the left Acom aneurysm was identified and clipped. The SSEP recordings remained unchanged during the process of temporary and post-clipping. The BP was maintained at the baseline values, and the noradrenaline infusion was gradually tapered and discontinued before extubation. The patient was extubated smoothly with a post-extubation GCS of $13 / 15$ without any neurological deficits and was transferred to the Neurosurgical Intensive Care Unit (ICU) with milrinone infusion which was later tapered and stopped in the ICU.

Within $2 \mathrm{~h}$ into the post-operative period, he developed signs of congestive heart failure manifesting as dyspnoea, hypotension $(90 / 50 \mathrm{mmHg})$, desaturation, bilateral basal crepitation of lungs and reduction in urine output. Immediate transthoracic echocardiography (TTE) was performed by the cardiologist, which revealed adequate pre-load and good myocardial contractility without any regional wall motion abnormality. The plasma concentration of $\mathrm{N}$-terminal pro-brain natriuretic peptide (NTpro-BNP) level was measured, which was significantly elevated $(3800 \mathrm{pg} / \mathrm{ml})$, suggesting the diagnosis of heart failure with preserved ejection fraction $(\mathrm{HFpEF})$. The milrinone infusion was initiated at $0.375 \mu \mathrm{g} / \mathrm{kg} / \mathrm{min}$ and increased to $0.5 \mu \mathrm{g} / \mathrm{kg} / \mathrm{min}$, the BP was restored to the baseline value and IV furosemide $20 \mathrm{mg}$ was administered and subsequently his urine output had improved within an hour. The milrinone infusion was continued for $24 \mathrm{~h}$ and then slowly tapered and stopped. Under intermittent TTE guidance, optimal IVF fluid was administered keeping the balance between the vasospasm and heart failure in mind. The patient recovered well without complications and was started on oral dabigatran and furosemide along with other routine medication and discharged on the $10^{\text {th }}$ post-operative day in a good clinical state.

\section{DISCUSSION}

Restrictive cardiomyopathy is the common cause of severe diastolic dysfunction. Diastolic heart failure is termed as HFpEF, and it is characterised by impairment in the distensibility of the myocardium, resulting in elevated ventricular diastolic pressures and thereby compromising the diastolic filling. ${ }^{[2,3]}$ Post-operative diastolic dysfunction is hard to diagnose and often challenging to manage. ${ }^{[4]}$ Due to lack of any definite clinical sign or specific test, diastolic failure is a diagnosis of exclusion and primarily depends on the combination of typical echocardiographic findings and elevated serum cardiac biomarkers. Echocardiographic findings are characterised by trans-mitral Doppler E/A >2 (early [E] to late [A] diastolic wave) and reduced early diastolic mitral annular tissue velocity $\left(\mathrm{e}^{\prime}\right)$ with a predominance of pulmonary venous ${ }^{\prime} \mathrm{D}^{\prime}$ wave amplitude. ${ }^{[2]}$ Although not specific, NTpro-BNP is the most relevant biomarker, which has been shown to be predictive of diastolic dysfunction. ${ }^{[2]}$ It is cleaved from the precursor hormone pro-BNP and released along with BNP, in response to the high ventricular tension. In a patient with clinical symptoms of shock, an elevated NTpro-BNP is highly suggestive of diastolic heart failure, provided systolic dysfunction is excluded by echocardiography. ${ }^{[1]}$ In our case, we have attained the clinical diagnosis using both echo finding and the NTpro-BNP value.

Maintenance of sinus rhythm, adequate filling pressures and avoidance of myocardial depression and an increase in afterload are the cornerstones in the perioperative management of diastolic dysfunction. Predisposing factors such as advanced age, the presence of hypertrophied myocardium, atrial fibrillation and post-operative ischaemia, all were implicated in the development of post-operative diastolic dysfunction. ${ }^{[5]}$ The risk of developing post-operative diastolic heart failure was very high in our patient because of the presence of multiple predisposing factors.

The ACC/AHA 2013 Class 1 recommendation for Stage III HFpEF is to maintain systolic and diastolic pressure with no specific mention of any agent. ${ }^{[2]}$ We selected milrinone, a selective phosphodiesterase-III inhibitor, because of the following reasons. (1) It promotes diastolic function with a concomitant reduction in pulmonary and systemic vascular resistance, without a considerable increase in heart rate and myocardial oxygen consumption ${ }^{[6]}$ and (2) It is less arrhythmogenic than dobutamine and therefore proved to be the most favourable inotropic agent. ${ }^{[7]}$

Utilisation of the TEE provided an accurate measurement of the pre-load and contractility and thereby enabled us in the appropriate management of the haemodynamics during the procedure. Furthermore, milrinone by its 
afterload-reducing property provides an additional advantage of counteracting the increased afterload associated with discontinuation of anaesthetic agents and post-operative stress. Retrospectively, we felt that early stopping of milrinone in the immediate post-operative period could have precipitated the diastolic heart failure. The timely initiation of milrinone infusion provided the most favourable haemodynamics for managing diastolic heart failure and averted further decompensation.

Furthermore, studies have documented a significant increase in cerebral perfusion pressure and improvement in global cerebral oxygenation using milrinone infusion in post-aneurysm clipping patients. ${ }^{[8]}$ It is one of the standard drugs used for the management of vasospasm following aneurysmal SAH.

\section{CONCLUSION}

We wish to conclude that the peri-operative utilisation of echocardiography (TEE and TTE) greatly facilitated the understanding of the underlying volume and contractile status of the heart and helped us to determine the appropriate intervention. Milrinone is the most suitable drug for managing acute post-operative diastolic failure in patients who undergo aneurysm clipping because of its 'dual beneficial' action, namely the cerebral vasodilatory effect, lusitropic and afterload-reducing properties, facilitating prompt recovery without any cardiac or neurological morbidity. Since patients with diastolic failure are extremely sensitive to even subtle variations in pre- and after-load, it would be prudent to avoid the loading dose of milrinone.

\section{Financial support and sponsorship} Nil.

\section{Conflicts of interest}

There are no conflicts of interest.

\section{REFERENCES}

1. Alsaddique AA. Recognition of diastolic heart failure in the postoperative heart. Eur J Cardiothorac Surg 2008;34:1141-8.

2. Gelzinis TA. New insights into diastolic dysfunction and heart failure with preserved ejection fraction. Semin Cardiothorac Vasc Anesth 2014;18:208-17.

3. Yancy CW, Jessup M, Bozkurt B, ButlerJ, Casey DE, Drazner MH, et al. 2013 ACC/AHA guideline for the management of heart failure. A report of the American College of Cardiology Foundation/American Heart Association task force on practice guidelines. Circulation 2013;128:e240-327.

4. Alsaddique AA, Royse AG, Royse CF, Fouda MA. Management of diastolic heart failure following cardiac surgery. Eur J Cardiothorac Surg 2009;35:241-9.

5. Albrecht CA, Giesler GM, Kar B, Hariharan R, Delgado RM $3^{\text {rd }}$. Intravenous milrinone infusion improves congestive heart failure caused by diastolic dysfunction: A brief case series. Tex Heart Inst J 2005;32:220-3.

6. Chatterjee K, De Marco T. Role of nonglycosidic inotropic agents: Indications, ethics, and limitations. Med Clin North Am 2003;87:391-418.

7. Anesthesia for Noncardiac Surgery in Patients with Heart Failure. Available from: http://www.uptodate.com/ contents/anesthesia-for-noncardiac-surgery-in-patientswith-heart-failure. [Last accessed on 2015 Dec 31].

8. Ghanem MA, Shabana AM. Effects of milrinone continuous intravenous infusion on global cerebral oxygenation and cerebral vasospasm after a cerebral aneurysm surgical clipping. Egypt J Anaesth 2014;30:73-82. 